\title{
Transient Response of Glycerin Heating Process
}

\author{
Zuriati Janin, Hazilah Mad Kaidi, Robiah Ahmad
}

\begin{abstract}
Controlling the temperature of the glycerin purification process system was not an easy task, as an increase in operating temperature would significantly reduce the quality of the purified glycerin. This is because an unlimited increase in temperature beyond the set point and an excessive prolongation of the heating process would result in the formation of an excessive secondary oxidation product in the final purified glycerin. This paper discusses the transient response characteristics of the glycerin heating process using a parallel PID controller. The glycerin heating process behavior was determined experimentally using step input test and modelled as the First Order plus Delay Time. The controller parameters wereadjusted using Ziegler-Nichols, Cohen-Coon and Wang tuning methods, each of which was analyzed on the basis of the corresponding integral error criterion value. The Integral Square Error, Integral Absolute Error and Integral Time-weighted Absolute Error criteria value were used to evaluate the efficiency of the glycerin heating process. The transient response performances in terms of overshoot, rise time and settling time were also evaluated. Simulation work has shown that the process has experienced high overshoots for Ziegler-Nichols and Cohen-Coon, and has taken longer time to settle. Wang method exhibits with no overshoot but slow response. The lower gain PID controller was found to improve the process response in terms of overshoot but increase in the rise time and settling time. The results indicate that the desired process performance were more or less influenced by the interaction between the tuning parameters. The Ziegler-Nichols PID controller is not recommended for controlling glycerin heating process due to process response oscillations that are difficult to eliminate without prolonging the heating cycle.
\end{abstract}

Keywords: Glycerin, Integral Error Criterion, PID Controller, Transient Response.

\section{INTRODUCTION}

The step response of the glycerin heating process systemis an important performance characteristic that determines the correctnessa system respond to the change in input temperature[1], [2].The proportional-integral-derivative (PID) controller is well known to still dominate most of the process industries [3], [4], [5]. This is because the PID controller is a very simple control system andcan be understood by non-experts [3], [6]. Until now, the PID

Revised Manuscript Received on December 30, 2019.

* Correspondence Author

Zuriati Janin*, Faculty of Electrical Engineering, Universiti Teknologi Mara, Shah Alam, Malaysia. E-mail: zuriaty@uitm.edu.my

Hazilah Mad Kaidi, Razak Faculty of Technology and Informatics, Universiti Teknologi, Malaysia, Kuala Lumpur, Malaysia. E-mail: hazilah.kl@utm.my

Robiah Ahmad, Razak Faculty of Technology and Informatics, Universiti Teknologi Malaysia, Kuala Lumpur, Malaysia. E-mail: robiahahmad@utm.my

(C) The Authors. Published by Blue Eyes Intelligence Engineering and Sciences Publication (BEIESP). This is an open access article under the CC BY-NC-ND license (http://creativecommons.org/licenses/by-nc-nd/4.0/) controller has remained relevant to the various system and has become a standard for the new design of the sophisticated controllers [7], [8], [9], [10], 11]. The objective of this paper is to scrutinize the implementation of the parallel PID controller for the glycerin heating process on the basis of the transient response characteristics and the corresponding integral error criterion value.

\section{GLYCERIN HEATING PROCESS}

\section{A. System Description}

In this work, the glycerin heating process is carried out in a closed tank in whichcrude glycerin is heated from the outside. In this arrangement, the heat transfer from the band heater is done usinga conductive system method.

As shown in Fig. 1, the heaters are appropriately calibrated and clamped around the outer diameter of the tank. The heaters are then covered with a fiber and aluminum foil to ensure that the heat from the heaters accumulates in the tank and holds the temperature steady. Band heaters in use work on the principle of resistive heating that an electrical current flowing through a conductor produces heat.

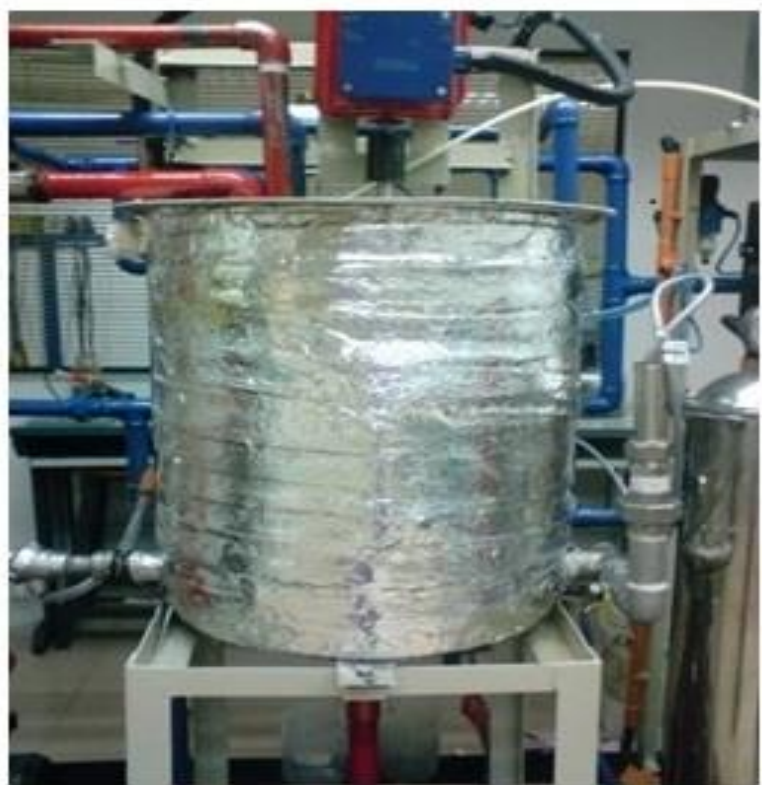

Fig. 1.Glycerin heating process system

\section{B. Control Design Consideration}

The glycerin heating process is modelled as first order plus delay time (FOPDT)as shown in (1) where $\tau, k$ and $t_{o}$ are process time constant, process gain and process time delay respectively. These process model parameters are obtained experimentally through an open loop step input response.

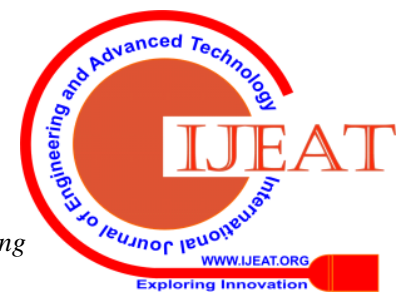




$$
G_{p}=\frac{k e^{-t_{o} s}}{\tau s+1}
$$

In this work, the temperature of the glycerin heating process needs to accelerate to the desired temperature of $85^{\circ} \mathrm{C}$ in a reasonable time with no overshoot in the process response. Furthermore, the system needs to maintain the temperature with a small deviation. The process flow for the completion of the study is as shown in Fig. 2.

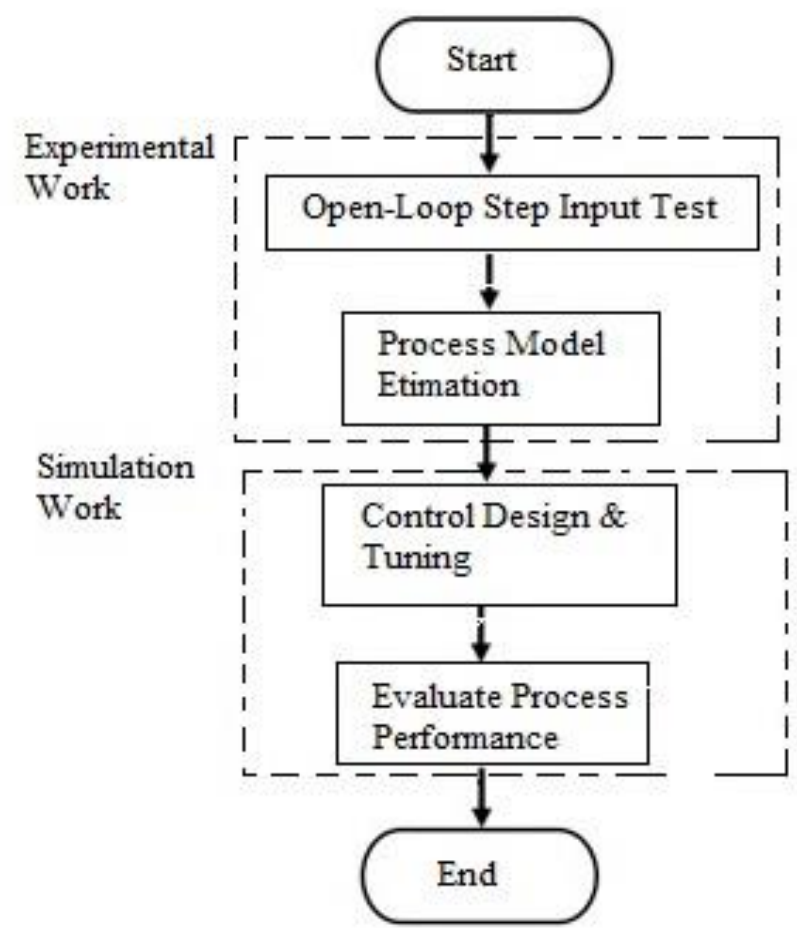

Fig. 2.Methodology process flow

\section{PID CONTROLLER AND TUNING METHODS}

\section{A. PID Controller}

In this work, a parallel PID controller in use is as shown in (2).

$$
u(t)=K_{p}\left(e(t)+\frac{1}{T_{i}} \int_{0}^{t} e(t) d t+T_{d} \frac{d e(t)}{d t}\right)
$$

The variable of $K p, T i$ and $T d$ shown in (2) are the controller parameters that need to be adjusted.

\section{B. Tuning Methods}

The Ziegler -Nichols tuning is the pioneer method in PID control system [12]. In this method, the controller parameters are adjusted according to the FOPDT process model. The proportional gain is determined as shown in (3). The integral and derivative time are determined using (4) and (5) respectively.

$$
\begin{gathered}
K_{p}=\frac{1.2 \tau}{k t_{o}} \\
T_{i}=2 t_{o}
\end{gathered}
$$

$$
T_{d}=0.5 t_{o}
$$

The Cohen -Coon tuning method for proportional gain is described in (6). Equation (7) and (8) are used to obtain the integral and derivative time.

$$
\begin{gathered}
K_{p}=\frac{\tau}{k t_{o}}\left(1+\frac{t_{o}}{3 \tau}\right) \\
T_{i}=\frac{\left(32 \tau+6 t_{o}\right) t_{o}}{13 \tau+8 t_{o}} \\
T_{d}=\frac{4 t_{o} \tau}{11 \tau+2 t_{o}}
\end{gathered}
$$

The proportional gain, integral time and derivative time for Wang tuning technique is calculated using (9), (10) and (11) respectively [13].

$$
\begin{gathered}
K_{p}=\frac{\left(0.7645+\frac{0.6032 \tau}{t_{o}}\right)}{k\left(\tau+t_{o}\right)}\left(\tau+0.5 t_{o}\right) \\
T_{i}=\tau+0.5 t_{o} \\
T_{d}=\frac{0.5 t_{o} \tau}{\tau+0.5 t_{o}}
\end{gathered}
$$

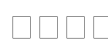

\section{PERFORMANCE EVALUATION}

In this work, the integral error criterion shown in (12), (13), and (14) are used to evaluate the performance of the controller.

$$
\begin{gathered}
I S E=\int_{0}^{t} e^{2} d t \\
I A E=\int_{0}^{t}|e| d t \\
\text { ITAE }=\int_{0}^{t} t|e| d t
\end{gathered}
$$
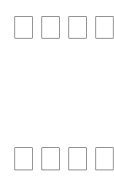

where

ISE = Integral Squared Error

$\mathrm{IAE}=$ Integral Absolute Error

ITAE = Integral Time-weighted Absolute Error

\section{RESULT AND DISCUSSION}

The controller parameters for all tuning methods are tabulated in Table-I.

Published By:

Blue Eyes Intelligence Engineering \& Sciences Publication 
Table- I: Controller parameters

\begin{tabular}{|c|c|c|c|}
\hline \multirow{2}{*}{ Tuning Methods } & \multicolumn{3}{|c|}{ Parameters } \\
\cline { 2 - 4 } & $\boldsymbol{K p}$ & $\boldsymbol{T i}$ & $\boldsymbol{T d}$ \\
\hline Ziegler-Nichols & 2.0 & 840 & 210 \\
\hline Cohen-Coon & 1.8 & 983 & 149.4 \\
\hline Wang Tuning & 1.1 & 3607 & 197.8 \\
\hline
\end{tabular}

The analysis is conducted in MATLAB R2017a environment in which the Simulink diagram for the process model is as shown in Fig. 3.

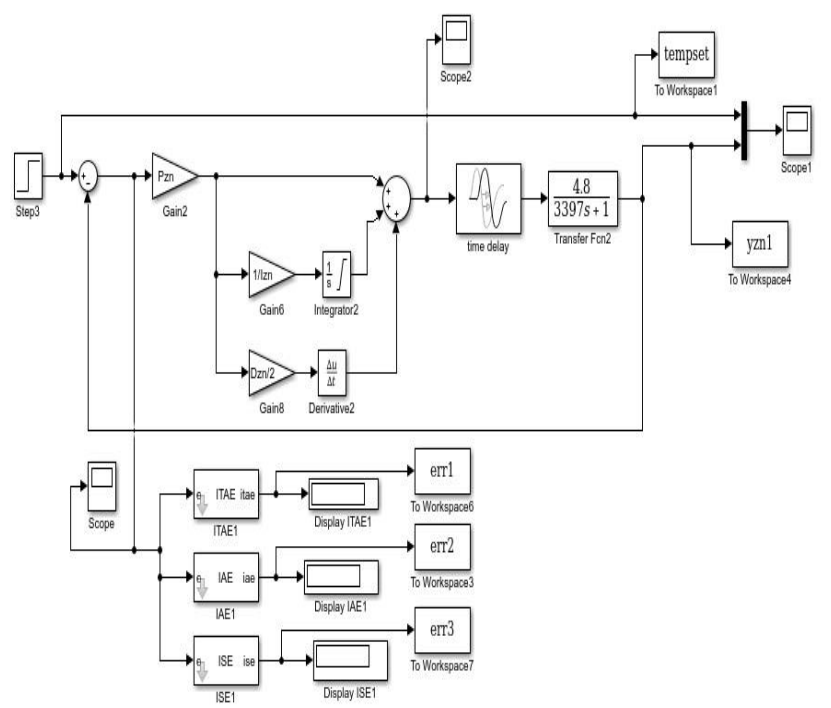

Fig. 3.Simulink diagram for analysis

Fig. 4 shows the closed loop step response of PID controller for the glycerin heating process. Obviously, it can be seen that Ziegler-Nichols PID exhibits high overshoot with oscillations response which can cause longer time to track the desired temperature.

Table II shows that the rise time for Ziegler-Nichols PID was quite fast as compared to the other methods but penalize with high overshoot of $60.4 \%$ deviates from the desired temperature. The overall settling time of the response is achieved at 7701.2 second. It can also be seen that Wang tuning method gave best result in term of percent overshoot.

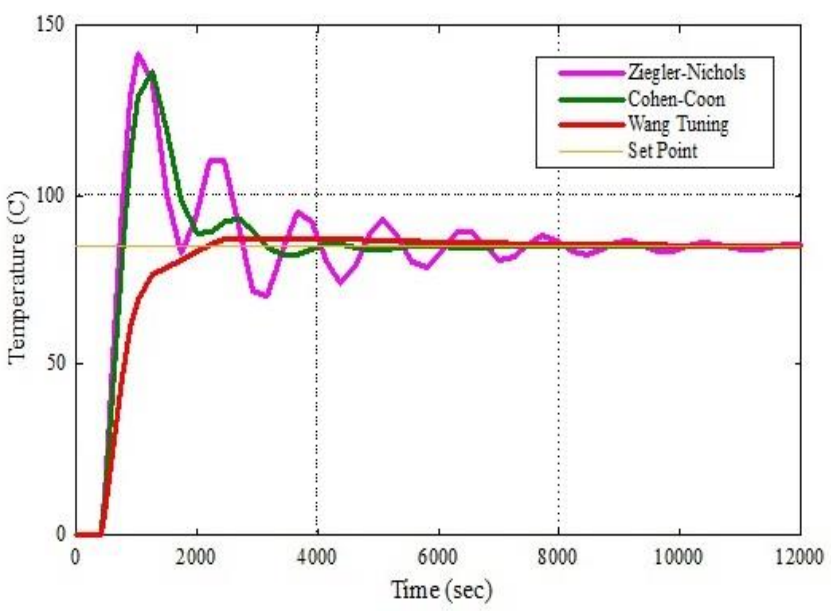

Fig. 4.PID controller performance comparison
Table- II: Transient response performance

\begin{tabular}{|c|c|c|c|}
\hline \multirow{2}{*}{$\begin{array}{c}\text { Tuning } \\
\text { Methods }\end{array}$} & \multicolumn{3}{|c|}{ Performances } \\
\cline { 2 - 4 } & $\begin{array}{c}\text { Rise } \\
\text { (sec) }\end{array}$ & $\begin{array}{c}\text { Settling } \\
\text { Time (sec) }\end{array}$ & $\begin{array}{c}\text { Oversho } \\
\text { ot (\%) }\end{array}$ \\
\hline $\begin{array}{c}\text { Ziegler-Nich } \\
\text { ols }\end{array}$ & 298.5 & 7701.2 & 60.4 \\
\hline Cohen-Coon & 385.6 & 5261.5 & 46.6 \\
\hline Wang Tuning & 664.8 & 5035.7 & 0 \\
\hline
\end{tabular}

The refined tuned controller performance is as shown in Fig. 5. The corresponding transient response characteristics are as shown in Table III.

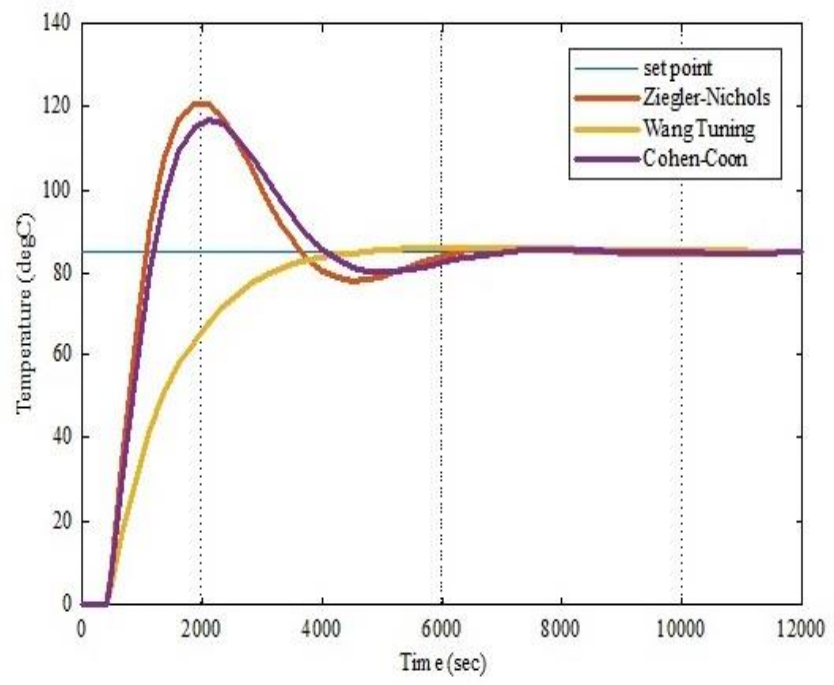

Fig. 5.PID controller performance comparison (refined tuned)

It can be seen in Fig. 5 that there is improvement in response behavior.Even though the rise time and the settling time of the controller are slightly increased, the overshoot of the closed loop controller has decreased.The overshoot of the closed loop PID Ziegler-Nichols decreased to $27.5 \%$ and the process response was settled at 7521.1 seconds. The process is seen approaching under damped performance response for Ziegler-Nichols and Cohen-Coon, in which the methods exhibit overshoot and increase the rise time. For the Wang tuning method, even though there is no overshoot presence at the output, the process took longer time to settle as compared to the other two methods.

The error plot at each point in time, t for all tuning methods is shown in Fig. 6. It can be seen that the controller error continually changes size and sign as time passes. There is area under the graph that developed over time if the response deviates from desired operating temperature. The integral error for all methods is tabulated in Table III. 


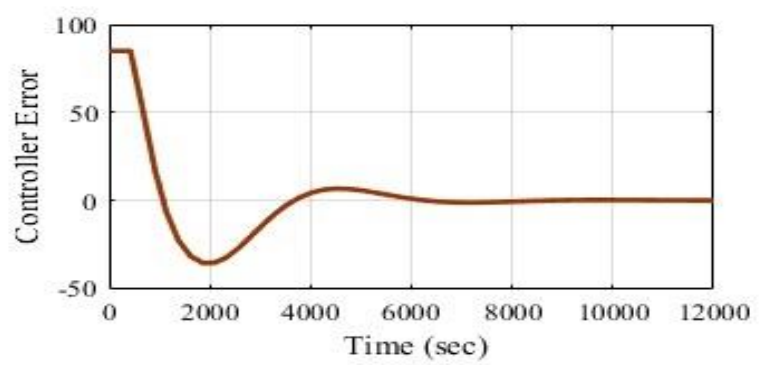

(a)

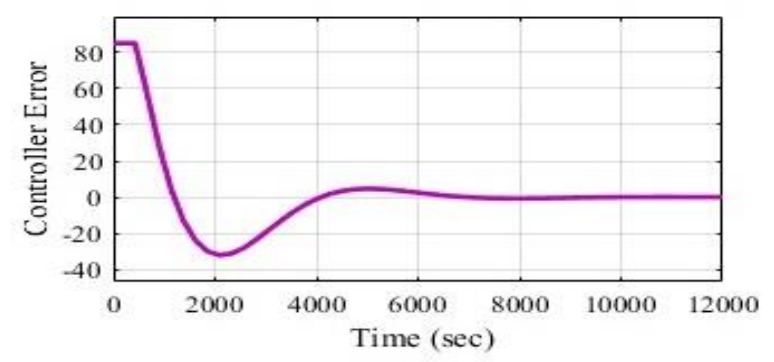

(b)

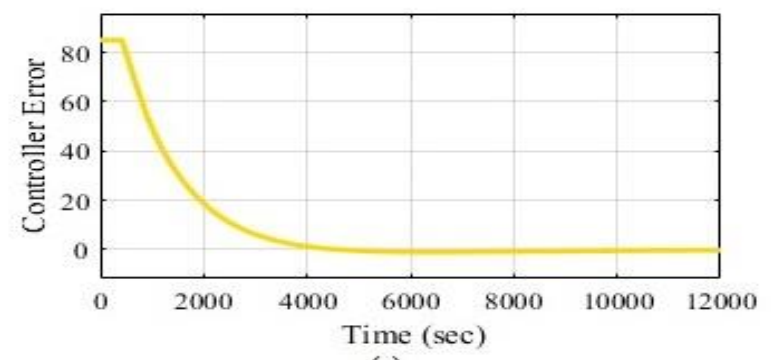

(c)

Fig. 6.Error plot (a) Ziegler-Nichols (b) Cohen-Coon (c) Wang
The error plot shows that when the ac power controller starts transmitting the electric current signal to the heater, the temperature starts to rise. As a result, the PID controller will stop the heater in order to get the temperature back to the set point. Due to the over-correctness and the complexity of the heating process, causing the temperature rate to undershoot the set point and oscillate a bit before finally settled.

Although only three parameters are involved in the adjustment of the PID controller, it is a challenging task for the glycerin heating process. This is because the interaction between the tuning parameters, more or less influencesthe desired purified glycerin.

\section{CONCLUSION}

The glycerin heating process transient response for PID controller is analyzed. It can be concluded that it is not an easy task to obtain the optimal controller for glycerin purification process with no overshoot and settle in short period of time without degrade the final purified glycerin. The PID controller with lower gain improves the transient response of glycerin heating process in term of overshoot but took longer time to settle.In conclusion, the PID controller which adjusted using Ziegler-Nichols method is not recommended for regulating the glycerin heating process because it produces high overshoot that is difficult to eliminate and prolongs the heating process.

Table- III: Overall glycerin heating process performance comparison

\begin{tabular}{|c|c|c|c|c|c|c|}
\hline & \multicolumn{2}{|c|}{ First Tuning } & \multicolumn{3}{c|}{ Refined Tuning } \\
\hline $\begin{array}{c}\text { Controller } \\
\text { Tuning } \\
\text { Method }\end{array}$ & Ziegler-Nichols & Cohen-Coon & Wang & Ziegler-Nichols & Cohen-Coon & Wang \\
\hline $\begin{array}{c}\text { Rise Time } \\
(\mathrm{sec})\end{array}$ & 298.5 & 385.6 & 664.8 & 561.8 & 592.2 & 1912.2 \\
\hline $\begin{array}{c}\text { Settling Time } \\
(\mathrm{sec})\end{array}$ & 7701.2 & 5261.5 & 5035.7 & 7521.1 & 7885.6 & 10120.0 \\
\hline Overshoot & 60.4 & 46.6 & 0 & 27.5 & 31.6 & 0 \\
\hline IAE $\left(10^{5}\right)$ & 1.30 & 0.96 & 0.83 & 1.32 & 1.31 & 1.26 \\
\hline ISE $\left(10^{5}\right)$ & 0.59 & 0.55 & 0.47 & 0.63 & 0.62 & 0.69 \\
\hline ITAE $\left(10^{5}\right)$ & 0.003 & 0.96 & 0.01 & 0.002 & 0.002 & 0.002 \\
\hline
\end{tabular}

\section{ACKNOWLEDGMENT}

Authors thank the Faculty of Electrical Engineering, Universiti Teknologi Mara (UiTM) and Faculty of Technology and Informatics, Universiti Teknologi Malaysia (UTM), Kuala Lumpur for their support to this research work.

\section{REFERENCES}

1. R. C. Dorf and R. H. Bishop, "PID Controllers in Modern Control Systems,” ch.7, p.502, M. J. Horton, Ed. New Jersey, USA: Pearson Education, Inc.,2011.

2. C. D. Johnson, Process Control Instrumentation Technology, Pearson / Prentice Hall, 2006.
3. J. J. Gude, E. Kahoraho and J. Etxaniz, Practical Aspects of PID Controllers: An Industrial Experience", in Proceeding of 2016 IEEE Conference on Emerging Technologies and Factory Automation, Prague, 2016.

4. A. O'Dwyer, "Pi and PID Controller Tuning Rules: An Overview and Personal Perspective," in Proceeding of Irish Signals and Systems Conference, 2006.

5. A. O'Dwyer, "Performance Improvement Using Simple PID Tuning Formulae," in Proceeding of the $3^{\text {rd }}$ IET International Conference on Power Electronics, Machines and Drives, Dublin, 2006.

6. A. O'Dwyer, "A Survey of Techniques for the Estimation and Compensation of Processes with Time Delay', 2000.

Published By:

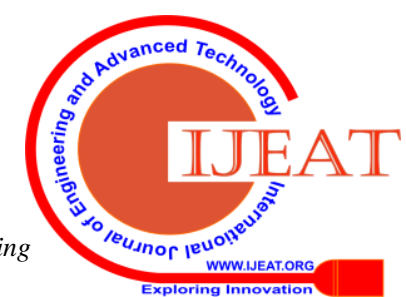


7. D. O. Aborisade and P. A. Adewuyi, "Evaluation of PID Tuning Methods on Direct Gas-Fired Oven", Int. Journal of Engineering Research and Applications, vol. 4, Issue 3 (ver. 1), pp. 01-09, 2014.

8. K. S. Chia, "Ziegler-Nichols Based Proportional-Integral-Derivative Controller for Line Tracking Robot”, Indonesian Journal of Electrical Engineering and Computer Science, vol.9, No. 1, pp. 221-226, 2018.

9. A. Daraz, S. Malik, T. Saleem and S. Bhati, "Ziegler-Nichols Based Integral Proportional Controller for Superheated Steam Temperature Control System," World Academy of Science, Engineering and Technology, International Journal of Electrical, Computer, Energetic, Electronic and Communication Engineering, vol. 11, No. 5, pp. 516-520, 2017.

10. B. M. Sarif, D. V. A. Kumar and M. V. G. Rao, "Comparison Study of PID Controller Tuning Using Classical/Analytical Methods", Int Journal of Applied Engineering Research, ISSN 0973-4562, vol. 13, No. 8, pp. 5618-5625, 2018.

11. E. Zambaldi, R. R. Magalhaes, B. H. Barbosa, S. P. d'Silva and D. D. Ferreira, "Low Cost Automated Control for Steel Heat Treatments", Applied Thermal Engineering, vol. 114, pp. 163-169, 2017.

12. G. Ziegler and N. B. Nichols, "Optimum Setting of Automatic Controllers", ASME Transaction, vol. 64, pp. 759-768, 1942.

13. W. Qing-Guo, L. Tong-Heng, F. Ho-Wang, B. Qiang and Z. Yu, "PID Tuning for Improved Performance", IEEE Transaction on Control System Technology, vol. 7, pp. 457-465, 19999.

\section{AUTHORS PROFILE}

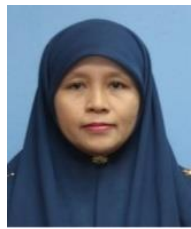

Zuriati Janin, received her B.Eng. in Electrical Engineering in 1996 and MSc. in Remote Sensing \& GIS from the Universiti Putra Malaysia (UPM) in 2001 and pursuing a Ph.D in Instrumentation and Control System at the Universiti Teknologi Malaysia (UTM). She has served as a SeniorLecturer at Universiti Teknologi Mara for more than 20 years at Faculty of Electrical Engineering, UiTM, Shah Alam. She has been involved with research in the area of control system, signal processing, instrumentation \& measurement, and engineering knowledge since 2004. She is a senior member of the Institute of Electrical and Electronics Engineers (IEEE) and was an Honorary Treasurer of IEEE Malaysia Section in 2016-2018.

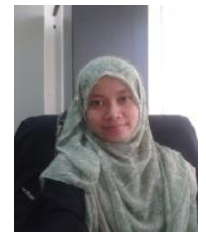

Hazilah Mad Kaidi received the B.Eng. (Horns) in Electrical Engineering (telecommunications), Universiti Teknologi Malaysia (UTM - 2006), the M.Sc. degree in Telecommunication and Information Engineering at Universiti Teknologi MARA (UiTM - 2008), and the Ph.D. degree from the Universiti Teknologi Malaysia (UTM - 2015). She is currently a senior lecturer at Razak Faculty of Technology and Informatics, Universiti Teknologi Malaysia Kuala Lumpur.She is a senior member of the Institute of Electrical and Electronics Engineers. She also a research member of Ubiquitous Broadband Access Network (U-BAN) research group and Wireless Communication Centre (WCC) which is one of the Higher Institution Centres of Excellence (HICoE) in Malaysia. Currently, WCC leads research on Fifth Generation (5G). Her research interests include mobile and wireless communications, Internet of Things, error control coding, relay networks, cooperative communications, Hybrid ARQ, Cross Layer Design and iterative receiver.

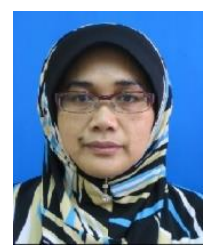

Robiah Ahmad received the B.Sc. (Electrical Engineering) in 1988, M.Sc. (Information Technology for Manufacture) in 1992 from WMG, University of Warwick, UK and Ph.D (Mechanical Engineering) in 2004 from Universiti Teknologi Malaysia (UTM). She is currently Associate Professor at Razak Faculty of Technology and Informatics, Universiti Teknologi Malaysia Kuala Lumpur. She is a senior member of the Institute of Electrical and Electronics Engineers. She also currently a Chair of IEEE Instrumentation and Measurement Society, IEEE Malaysia Section. She had published 83 indexed publications since 2012. Her research interests include System Identification and Modelling, Evolutionary Computation, Control Systems Engineering, Instrumentation and Measurement System, LEDAR Lab, Agriculture and LED Lights. 